\title{
Lower thoracic disc herniation mimicking lower lumbar disk disease: A case report
}

\author{
Mohamadreza Golbakhsh ${ }^{1}$, Arash Mottaghi ${ }^{2 *}$, Mohamreza Zarei $^{3}$ \\ Received: 12 Dec 2016 \\ Published: 16 Dec 2017
}

\begin{abstract}
Thoracic disk herniation comprises $0.15 \%$ of all disk herniation and has various and confusing manifestations. Among them, radicular pain down the leg could be the rarest presentation, especially if it is the only complaint. On the other hand, finding the relationship between clinical and paraclinical needs require high index of suspension and it is demanding. A 34-year-old patient, who had a history of intermittent back pain, with lower thoracic disk herniation presented by acute leg sciatica-like pain, is reported. He suffered 3 weeks of acute back pain prior to admission, which radiated down to buttock and leg, with a vague left abdominal pain, whose clinical examination indicated a distal lumbar problem. MRI showed T-12 L-1 disk herniation. Lower thoracic disc herniation can compress lumbosacral roots immediately after exiting from cord thickening in the lower thoracic area, so they can incite lower lumbar radiculopathy and cause discordance between MRI findings and clinical presentation, suggesting a lumbar problem, and this can lead to delayed diagnosis. However, the acute pain was completely improved after open discectomy.
\end{abstract}

Keywords: Thoracic disc herniation, Leg radiculopathy, Lumbar disk herniation

Copyright $\subseteq$ Iran University of Medical Sciences

Cite this article as: Golbakhsh M, Mottaghi A, Zarei M. Lower thoracic disc herniation mimicking lower lumbar disk disease. Med J Islam Repub Iran. 2017 (16 Dec);31:87. https://doi.org/10.14196/mjiri.31.87

\section{Introduction}

Symptomatic thoracic disk diseases are uncommon, and their clinical manifestation differs widely from more common cervical and lumbar disk herniation to the well-known root compression (1). They account for approximately $0.15 \%$ of all disk herniations, and their peak incidence is in the fourth decade of life (2). Among the symptoms, leg radiculopathy, or sciatica-like pain are not the major complaints, whereas cord compression symptoms and myelopathy and sometime a band-like radicular component are more common (3). Cord compression can cause burning or tract dorsal pain (Lhermitte sign) below the lesion and gets worse by spine rotation due to irritation of ascending spinothalamic tract. Also, a dorsal vertebral pain is a vague and diffuse pain and resembles normal population back pain and fails to conform to any dermatomal distribution. Acute and subacute thoracic disc herniation occur in less than $10 \%$ of patients (4). Typically, it is the consequence of a soft herniation and is usually preceded by a minor trauma. A progressive course over a month or a year is predominant and calcification develops in more than $90 \%$ of patients (5). We present an unusually lower thoracic disk case with symptoms that correspond to lumbar disc disease.
Among the symptoms, radicular pain down the leg could be the rarest presentation, especially if it is the only complaint. On the other hand, finding the relationship between clinical and paraclinical needs require high index of suspension and it is demanding because some patients have degenerated $\mathrm{T} 12-\mathrm{L} 1$ as a false positive paraclinical finding.

\section{Case report}

A 34- year- old male with 3 weeks of acute left sciaticalike pain down to calf and concomitant dorsal pain and a vague left abdominal pain referred to our hospital. He has already undergone conservative treatment in other facilities, however, no improvement occurred. He had a history of intermittent back pain 2 years prior to his admission. Pain was worsened by any respiratory movement, including coughing, sitting, and a prone position, but relieved by rest and upright lordotic position. The patient also complained of paresthesia in the dorsolateral calf and plantar surface area of the foot. There was also a complaint of voiding difficulty. On inspection, we found that he had paravertebral muscle spasm and some spine list. Lasegue manoeuver on 

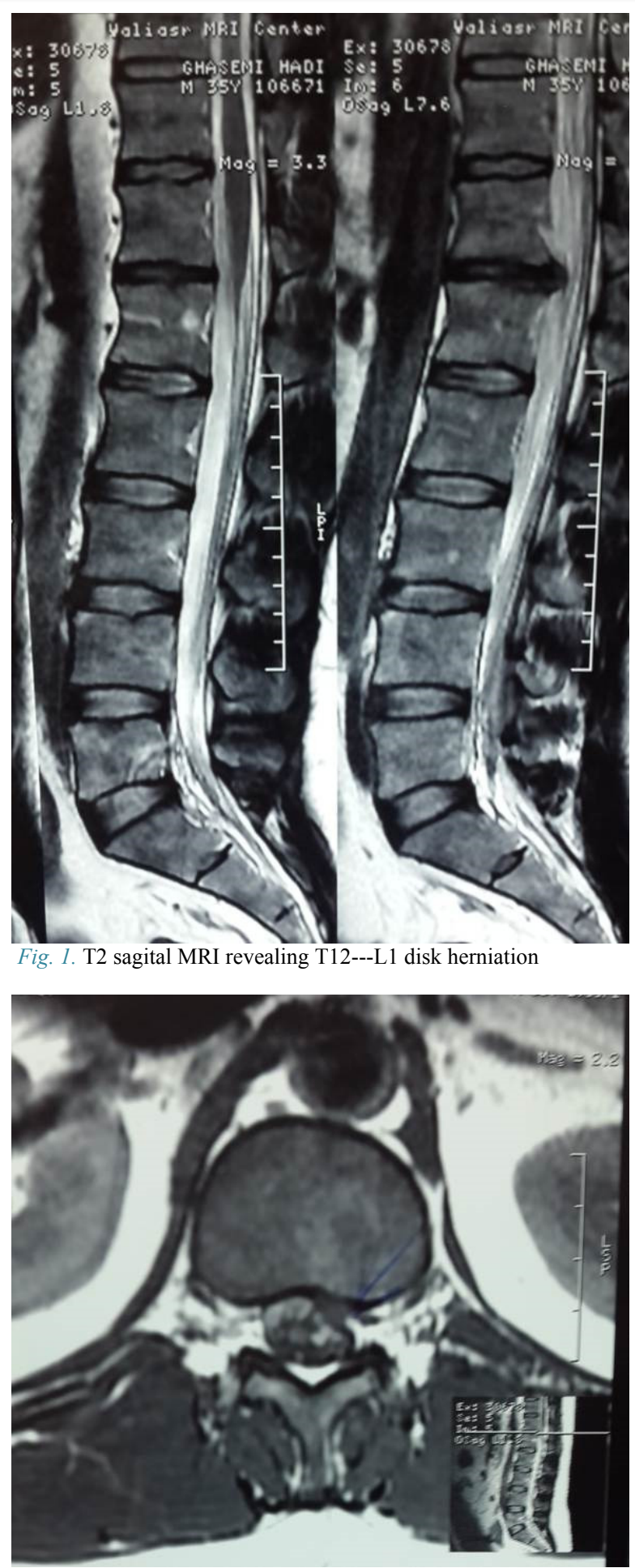

Fig. 2. Representing a postero-lateral soft disk herniation with its coresponding compression on conus

either side provoked right sided pain. Fine neurological examination revealed a band of slight hyposthesia in the T10 level, approximately. In the lower extremity, there was a doubtful increase in the knee and ankle jerk; great toe flexion and extension and ankle dosiflexion power was Grade 3,3 , and 4 , respectively, out of 5 . No pathological reflexes like babanski were observed. Standard x-rays of thoracolumbar area were normal. EMG-NCV showed some dysfunction in the pathway of L5 and S1 roots. MRI demon-

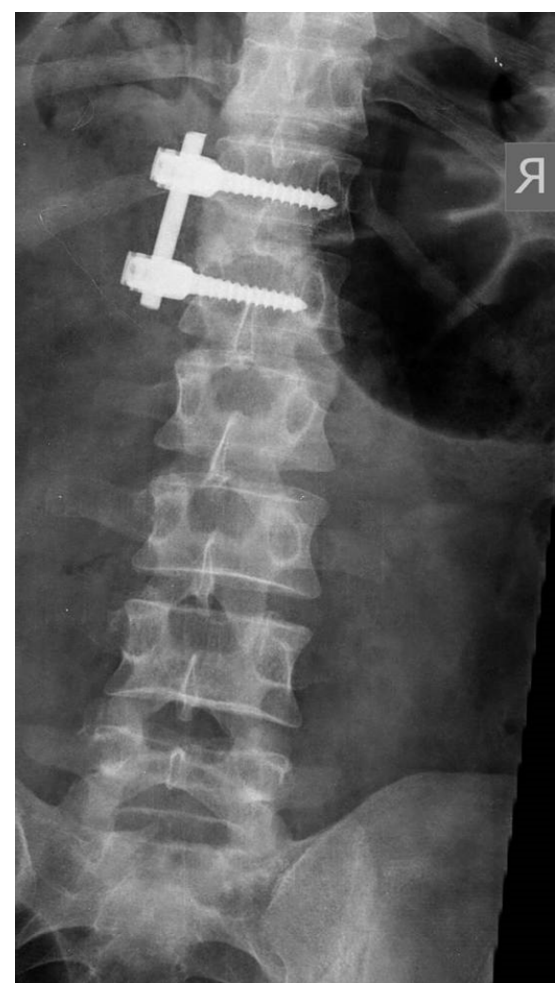

Fig. 3. Standard X-ray taken after discectomy and fusion by a left thoracotomy

strated a left posterolateral non-calcified T12-L1 disk herniation, with compression on conus medularis (Figs. 1 and 2). Also, the prominent acute manifestation in our patient suggested a lumbar pathology. However, after delicate history taking and fine examination, we scheduled a left-sided thoracotomy and discectomy and fusion (Fig. 3). Soon after the operation, the radicular pain disappeared in the patient. On the 6-month follow-up, it was found that the normal forces were recovered and voiding frequency was improved in the patient. Normal senses were observed after 14 months. However, mild lower back pain persisted on the 2year follow-up due to disk degenerative diseases.

\section{Discussion}

Thoracic disk herniation is not frequently encountered compared to corresponding lumbar and cervical herniation. Proper diagnosis is usually delayed or sometime overlooked due to confusing presentations. Most commonly, herniation manifests with progressive myelopathy, flaccid paralysis, dorsal burning pain below the lesion (Lhermitte symptom), which is worsened by spine rotation and a vague and diffuse dorsal pain that mimics the normal back pain (6). However, sciatica-like pain or radiation of pain to buttock and leg is extremely rare, and thus may lead the surgeon to misdiagnose the condition, especially after a rush examination and history taking. On the other hand, some patients have a false positive degenerated disk in junction. This radicular pain is because of lumbosacral root compression by lower thoracic disk herniation after they exit from the enlargement of spinal cord before conus. The intradural roots in this place follow a highly organized arrangement with the rostral roots laterally and caudal ones centrally (7). 
Thus, a central disk herniation in lower thoracic area is sufficient to cause sciatica-like pain. Because the noteworthy symptom was sciatica-like pain in our patient, apt diagnosis was delayed and further workup was pursued after nonresponsiveness to conservative treatment. Because the MRI finding did not justify the clinical picture in our patient, we proceeded with dorsal MRI, which revealed the major culprit. Moreover, a band hyposthesia in the approximate level of T9, increased knee, and ankle jerk were found in examination. Some disturbances in voiding and left abdominal pain were the other clues to proper diagnosis and performing an appropriate treatment. Therefore, in every patient with unusual presentation and disharmony between clinical and paraclinical needs, careful history taking and painstaking neurological examination are essential to reach an accurate diagnosis and discover the cause and effect.

\section{Conflict of Interests}

The authors declare that they have no competing interests.

\section{References}

1. Cho HL, Lee SH, Kim JS. Thoracic disk herniation manifesting as sciat ica-like pain--two case reports.. Neurol Med Chir (Tokyo). 2011;51(1): 67-71.

2.Zhuang QS, Lun DX. Surgical Treatment for Central Calcified Thoraci c Disk Herniation. Orthopedics. 2015 Sep;38(9):e794-8.

3. Lara FJ, Berges AF. Thoracic disk herniation, a not infrequent cause of chronic abdominal pain. Int Surg. 2012 Jan-Mar;97(1):27-33.

4.Ziewacz JE, Mummaneni P. A novel approach to thoracic disk herniati on. World Neurosurg. 2013 Sep-Oct;80(3-4):317-318.

5. Bydon M, Gokaslan Z. Minimally invasive approaches in the treatment of thoracic disk herniation. World Neurosurg. 2014 May-Jun;81(5-6):7 17-718.

6. Miyakoshi N, Hongo M. Thoracic disk herniation with hematoma-case report. Neurol Med Chir (Tokyo). 2008 Sep;48(9):414-417.

7. Giblin EM, Hochheiser GM. Thoracic disk herniation resulting in acute ly progressing paraplegia in a pediatric patient Pediatr Emerg Care. 200 8 Aug;24(8):550-553. 